\title{
Feynman-Hellmann approach to the spin structure of hadrons
}

\author{
A. J. Chambers, ${ }^{1, *}$ R. Horsley, ${ }^{2}$ Y. Nakamura, ${ }^{3}$ H. Perlt, ${ }^{4}$ D. Pleiter, ${ }^{5,6}$ P. E. L. Rakow, ${ }^{7}$ G. Schierholz, ${ }^{8}$ \\ A. Schiller, ${ }^{4}$ H. Stüben, ${ }^{9}$ R. D. Young, ${ }^{1}$ and J. M. Zanotti ${ }^{1}$ \\ (CSSM and QCDSF/UKQCD Collaborations)
}

\begin{abstract}
${ }^{1}$ CSSM, Department of Physics, University of Adelaide, Adelaide, South Australia 5005, Australia
${ }^{2}$ School of Physics and Astronomy, University of Edinburgh, Edinburgh EH9 3JZ, United Kingdom

${ }^{3}$ RIKEN Advanced Institute for Computational Science, Kobe, Hyogo 650-0047, Japan

${ }^{4}$ Institut für Theoretische Physik, Universität Leipzig, 04103 Leipzig, Germany

${ }^{5}$ JSC, Jülich Research Centre, 52425 Jülich, Germany

${ }^{6}$ Institut für Theoretische Physik, Universität Regensburg, 93040 Regensburg, Germany

${ }^{7}$ Theoretical Physics Division, Department of Mathematical Sciences, University of Liverpool, Liverpool L69 3BX, United Kingdom

${ }^{8}$ Deutsches Elektronen-Synchrotron DESY, 22603 Hamburg, Germany

${ }^{9}$ Regionales Rechenzentrum, Universität Hamburg, 20146 Hamburg, Germany
\end{abstract}

(Received 22 May 2014; published 24 July 2014)

\begin{abstract}
We perform a $N_{f}=2+1$ lattice QCD simulation to determine the quark spin fractions of hadrons using the Feynman-Hellmann theorem. By introducing an external spin operator to the fermion action, the matrix elements relevant for quark spin fractions are extracted from the linear response of the hadron energies. Simulations indicate that the Feynman-Hellmann method offers statistical precision that is comparable to the standard three-point function approach, with the added benefit that it is less susceptible to excited-state contamination. This suggests that the Feynman-Hellmann technique offers a promising alternative for calculations of quark line disconnected contributions to hadronic matrix elements. At the SU(3)-flavor symmetry point, we find that the connected quark spin fractions are universally in the range 55\%-70\% for vector mesons and octet and decuplet baryons. There is an indication that the amount of spin suppression is quite sensitive to the strength of SU(3) breaking.
\end{abstract}

DOI: $10.1103 /$ PhysRevD.90.014510

PACS numbers: $12.38 . \mathrm{Gc}, 14.20 .-\mathrm{c}$

\section{INTRODUCTION}

The decomposition of the nucleon spin presents a fascinating challenge for the theoretical understanding of nonperturbative QCD. While the simplest quark model has all of the nucleon spin attributed to the spin of its quark constituents, the latest experimental measurements suggest that only about one third of the nucleon spin comes from the spin of the quarks [1]. This has motivated an extensive theoretical effort to understand the QCD origins of this quark spin suppression. We refer the reader to the comprehensive reviews of Refs. [2-5].

Lattice QCD provides a systematically improvable technique to study nonperturbative features of QCD, and hence offers significant potential to give valuable insight into the spin decomposition of the nucleon. Recent results have been published in [6-9] —also see the lattice review [10]. Nevertheless, there are still challenges in the lattice formulation, particularly those associated with the simulation of so-called "disconnected" quantities. Disconnected quantities refer to those where the external probe couples to a hadron correlator only through the underlying gauge

"alexander.chambers@adelaide.edu.au field configuration. Standard three-point function techniques require the stochastic estimation of these quark loop contributions and, while progress has been made, e.g. $[11,12]$, it has proven to be notoriously difficult to extract a nonzero signal.

In the present paper we explore an alternative technique for the extraction of hadronic spin matrix elements in lattice QCD. In particular, we utilize the Feynman-Hellmann (FH) theorem applied to the lattice regularization framework. We consider the energy shifts of hadrons in the presence of a uniform weak external field which couples directly to the quark spin. This is similar to an idea proposed in [13]. By the FH theorem, the leading linear response of the energy can be identified with the corresponding spin matrix element of interest. A first exploration of this method was performed in [14], and later in [15], for the gluon energy-momentum tensor. A full simulation would require the generation of new gauge ensembles which modify the fermion action of the sea quarks, incorporating the external field. Here we establish the method by coupling the field to the connected quark fields and benchmark our results against standard three-point function techniques.

There are some key advantages of the FeynmanHellmann method. Importantly, there has been plenty of 
debate surrounding the difficulty in controlling excitedstate contamination in conventional three-point function calculations of $g_{A}$ [16-21]. Since the FH method outlined in this paper only requires the extraction of hadron energies from lattice two-point functions, greater control of excitedstate contamination is possible through the identification of a distinct effective mass plateau. In addition, the quark propagators generated in the presence of the external field can be inserted into any hadronic correlation function and therefore, for a single set of inversions, one can study the spin content of many different hadrons. In contrast, usual three-point function methods require a new sequential propagator for each hadronic state of interest. ${ }^{1}$

With easy access to a variety of hadronic states, we are able to report first dynamical lattice QCD simulation results for the spin content of vector mesons and decuplet baryons, in addition to the baryon octet. Interestingly we find that at the SU(3)-flavor symmetric point of our simulations the connected quark spin fraction is around 55\%-70\%, irrespective of the hadron in question. This is in line with the general expectation of relativistic corrections to quark model wave functions [22-24]. We also present results away from the $\mathrm{SU}(3)$ symmetric point, where we find SU(3) breaking effects that could lead to significant breakdown of this universality in the light-quark domain [25].

The outline of the manuscript is as follows. Section II describes the formalism and notation used in this paper, and the strategy for the implementation of the FeynmanHellmann theorem in lattice QCD simulations (a detailed derivation of the theorem is included in Appendix A). The lattice configurations of the present study are reviewed in Sec. III, and the analysis techniques are described in Sec. IV. Our numerical results for various hadrons are reported in Sec. V. Concluding remarks are summarized in Sec. VI.

\section{FORMALISM}

In this section we present the formalism and notation used in this paper with regard to the spin structure of hadrons, and explain the approach of using the FeynmanHellmann theorem to calculate matrix elements.

\section{A. Spin notation}

We express the total spin of a hadron of spin $J$ by

$$
J=\frac{1}{2} \Delta \Sigma^{J}+L_{q}^{J}+J_{G}^{J},
$$

where $L_{q}^{J}$ and $J_{G}^{J}$ denote the quark orbital angular momentum and gluon angular momentum, respectively. The total quark spin sum is given by $\Delta \Sigma^{J}=\sum_{q} \Delta q^{J J}$, which in the

\footnotetext{
${ }^{1}$ Nevertheless, the standard three-point method can access many different matrix elements simultaneously for a given choice of hadronic state.
}

Bjorken limit is defined in terms of the zeroth moments of the polarized quark distributions,

$$
\Delta q^{J m}=\int \mathrm{d} x\left[q_{\uparrow}^{J m}(x)-q_{\downarrow}^{J m}(x)\right] .
$$

Our notation is such that these describe generalizations of polarized quark distributions for hadrons of spin $J$ with longitudinal spin polarization $m$, as defined by Ref. [26]. In lattice simulations, these can be computed by evaluating matrix elements of the local operator

$$
A_{q}^{\mu}=\bar{q} i \gamma_{5} \gamma^{\mu} q .
$$

In the rest frame of the hadron, the forward matrix elements of this operator directly isolate the quark spin contributions,

$$
\left\langle H, J m\left|A_{q}^{3}(0)\right| H, J m\right\rangle=2 M_{H} \Delta q^{J m},
$$

for a hadron with polarization $m$ with respect to the $z$ axis. It is these matrix elements that we wish to determine for a variety of spin- $J$ hadrons, $H$.

\section{B. The Feynman-Hellmann method}

The Feynman-Hellmann theorem offers an alternative method for calculating matrix elements of a particular operator. In Appendix A we derive the theorem as relevant for lattice calculations. Here we summarize the main points.

Suppose we wish to calculate the matrix element of an operator $\mathcal{O}$ with some hadron state $|H\rangle$. Consider modifying the QCD action such that

$$
S \rightarrow S+\lambda \int \mathrm{d}^{4} x \mathcal{O}(x)
$$

where $\lambda$ is a real parameter, and $\mathcal{O}$ is a local operator. Then by the Feynman-Hellmann theorem we have that

$$
\frac{\partial E(\lambda)}{\partial \lambda}=\frac{1}{2 E(\lambda)}\langle H|\mathcal{O}| H\rangle_{\lambda},
$$

where $E$ is the energy of the hadron state, and the subscript $\lambda$ on the correlator indicates that it is evaluated with respect to the modified action. Note when $\lambda=0$, we have

$$
\left.\frac{\partial E(\lambda)}{\partial \lambda}\right|_{\lambda=0}=\frac{1}{2 E}\langle H|\mathcal{O}| H\rangle,
$$

where the matrix element on the right-hand side is now with respect to the unmodified action. If the modification in Eq. (5) is made in the context of a lattice calculation, then one can examine the behavior of hadron energies as the parameter $\lambda$ changes, and extract the above matrix element at the point where $\lambda=0$. 
Recall the lattice estimate of the expectation value of an operator $\mathcal{O}$ over field configurations $U^{(i)}$ is given by

$$
\langle\mathcal{O}\rangle \approx \frac{1}{N} \sum_{i=1}^{N} \overline{\mathcal{O}}\left[U^{(i)}\right]
$$

where the bracket over $\mathcal{O}$ indicates that all quark bilinears in $\mathcal{O}$ have been Wick contracted and replaced with quark propagators, and where the field configurations have been generated using the weighting

$$
\operatorname{det}[D(U)] e^{-S_{g}[U]} .
$$

There are two points at which modifications to the action may be made in this calculation.

Firstly, quark propagators in the operator $\mathcal{O}$ are calculated by inverting the Dirac operator matrix. This matrix is given by the quark contribution to the QCD action, and so must be modified if we change the quark action. This change is straightforward to apply, only requiring a redefinition of the Dirac operator.

Secondly, we note that the weighting of the gauge fields in Eq. (9) depends on both the quark component of the action in the functional determinant, and the gluon component in the exponential. Hence, any modification we make to the action should be included during the generation of the background gauge fields.

By choosing to neglect either one of these modifications, we are able to individually isolate connected and disconnected contributions to matrix elements. Modifications to the gauge configurations allow access to disconnected quantities, and modifications to the calculation of propagators allow access to connected quantities.

The method above presents several advantages for calculating, in particular, quantities such as the disconnected quark contributions to the proton spin. Such disconnected contributions are included in a simulation during the generation of gauge configurations, and the calculation of the appropriate matrix element is reduced to the calculation of hadron energies for different values of $\lambda$, in order to apply Eq. (7).

We will demonstrate the implementation of the FeynmanHellmann method by calculating the connected quark contributions to the spin of hadrons. This has been investigated previously using standard three-point function methods, results with which we will compare our calculations.

The simulations discussed in Secs. IV and V make use of the partially quenched case for calculating connected quantities, and we do not generate any modified field configurations in the present paper.

\section{SIMULATION DETAILS}

We use gauge field configurations with $2+1$ flavors of nonperturbatively $\mathcal{O}(a)$-improved Wilson fermions and a lattice volume of $L^{3} \times T=32^{3} \times 64$. The lattice spacing $a=0.074(2) \mathrm{fm}$ is set using a number of singlet quantities [27-29]. The clover action used comprises the tree-level Symanzik improved gluon action together with a stout smeared fermion action, modified (as described in Sec. IV) for the implementation of the Feynman-Hellmann method. We have ensembles with two sets of hopping parameters, $\left(\kappa_{l}, \kappa_{s}\right)=(0.120900,120900),(0.121040,120620)$, where we work in the isospin-symmetric limit such that $\kappa_{l}=$ $\kappa_{u}=\kappa_{d}$. Table I gives the masses of various hadrons as realized on these configurations [29].

As discussed in the next section, the initial investigation of this method is performed at the $\mathrm{SU}(3)$ symmetric point $\left(\kappa_{l}=\kappa_{s}=0.120900\right)$ where all three quarks have the same mass, corresponding to a pion mass of around $470 \mathrm{MeV}$. On a subset of 350 configurations we explore the feasibility of the method using up to four different values of $\lambda$.

After tuning the method at this point, we then apply it to an ensemble with a lighter pion mass of around $360 \mathrm{MeV}$. As all of our lattice ensembles are generated with the singlet quark mass $\bar{m}=\frac{1}{3}\left(2 m_{l}+m_{s}\right)$ held fixed, this lattice also contains a heavier strange quark. This will allow us to demonstrate the suitability of this method for the study of the quark spin contributions to a variety of hadrons.

Unless otherwise stated, all results quoted in the remainder of this paper are unrenormalized (indicated by a superscript "latt."). However, in order to compare with existing results in the literature, we use preliminary results for the nonsinglet axial current renormalization constant [30]

$$
Z_{A}^{\mathrm{NS}}=0.85(2) .
$$

We note that most of the results quoted in the remainder of the paper are either for the total or individual quark spin contributions to a hadron's spin which also requires knowledge of the singlet axial current renormalization $Z_{A}^{S}$ [11], which has an anomalous dimension. Since $Z_{A}^{S}$ deviates from $Z_{A}^{\mathrm{NS}}$ starting at $\mathcal{O}\left(\alpha_{S}^{2}\right)$ in perturbation theory, we expect $Z_{A}^{\mathrm{S}}$ in the $\overline{\mathrm{MS}}$ scheme at $\mu^{2}=4 \mathrm{GeV}^{2}$ to differ from $Z_{A}^{\mathrm{NS}}$ by no more than a couple of percent. A similar sized correction maybe be needed to achieve full $\mathcal{O}(a)$ improvement [31]. In future work where we intend to also include disconnected contributions, we will implement a proper treatment of the

TABLE I. Table of hadron masses (in lattice units) for each ensemble.

\begin{tabular}{lll}
\hline \hline$\kappa_{l}$ & 0.120900 & 0.121040 \\
$\kappa_{s}$ & 0.120900 & 0.120620 \\
\hline$a M_{\pi}$ & $0.1747(5)$ & $0.1349(5)$ \\
$a M_{N}$ & $0.4673(27)$ & $0.4267(50)$ \\
$a M_{\Lambda}$ & $0.4673(27)$ & $0.4547(43)$ \\
$a M_{\Delta}$ & $0.5676(64)$ & $0.5520(79)$ \\
$a M_{\rho}$ & $0.3341(34)$ & $0.3127(38)$ \\
\hline \hline
\end{tabular}


renormalization. However, for the exploratory work carried out in this paper, we neglect these minor corrections and simply use $Z_{A}^{\mathrm{NS}}$ in Eq. (10) when a comparison of renormalized results is made.

\section{ANALYSIS TECHNIQUES}

Here we show how the Feynman-Hellmann theorem may be applied to calculate quark axial charges of hadrons, using the proton as an example. We will then show how the determination of these axial charges can be improved through the use of ratios of lattice two-point functions. Finally we will investigate the optimal choice of $\lambda$ values needed to reliably determine the axial charges at minimal computational cost.

\section{A. Spin operator and spin projection}

In our simulations, we modify the QCD action such that

$$
S \rightarrow S(\lambda)=S+\lambda \sum_{x} \bar{q}(x) i \gamma_{5} \gamma_{3} q(x),
$$

where $q$ denotes a particular quark flavor. Note $i \gamma_{5} \gamma_{3}$ is the Euclidean-space form of the spin operator in the $z$-direction. By application of the Feynman-Hellmann theorem for a zero-momentum hadron $H$ we have

$$
\left.\frac{\partial E(\lambda)}{\partial \lambda}\right|_{\lambda=0}=\frac{1}{2 M}\left\langle H\left|\bar{q} i \gamma_{5} \gamma_{3} q\right| H\right\rangle .
$$

Comparing with Eq. (3), we see that this slope gives direct access to the quark spin contributions,

$$
\Delta q=\left.\frac{\partial E(\lambda)}{\partial \lambda}\right|_{\lambda=0} .
$$

For simplicity, we have suppressed the explicit $J$ and $m$ spin indices, as is conventional for a spin- $\frac{1}{2}$ target. Calculation of $\Delta q$ has now been reduced from the calculation of lattice three-point functions to the simpler task of measuring energies from lattice two-point functions.

In our simulations, the modification to the action in Eq. (11) is only made to the Dirac matrix when calculating propagators, hence we only access the quark connected contributions to $\Delta q$, as discussed in Sec. II. Hence on the lattice, we have that

$$
\Delta q_{\mathrm{conn}}^{\mathrm{latt}}=\left.\frac{\partial E(\lambda)}{\partial \lambda}\right|_{\lambda=0} .
$$

Calculation of proton energies proceeds via normal lattice hadron-spectroscopy techniques. We make use of the standard proton interpolating operator

$$
\mathcal{O}_{p}=\epsilon_{a b c}\left(u_{a}^{T} C \gamma_{5} d_{b}\right) u_{c},
$$

where only color indices are shown explicitly; spinor indices are implied by matrix and vector notation. We use the positive parity projection operator (in Euclidean space)

$$
\Gamma_{4}=\frac{1}{2}\left(1+\gamma_{4}\right)
$$

to project out the positive parity state. Since the matrix element in Eq. (3) requires the hadron state to have definite spin, we combine the operator in Eq. (16) with spinprojection operators,

$$
\Gamma_{ \pm}=\frac{1}{2}\left(1 \pm i \gamma_{5} \gamma_{3}\right) \Gamma_{4} .
$$

Together these operators allow us to project out the $m= \pm \frac{1}{2}$ positive-parity proton states.

Recalling Eq. (11), we note that reversing the spin polarization of the hadron state is equivalent to reversing the sign of $\lambda$. Hence with a single choice of $\lambda$ we are able to identify the energies of the spin-up proton with positive $\lambda$, and those of the spin-down proton with negative $\lambda$. In this way, we effectively double our sampled parameters, without increasing the simulation time.

As a first test, we simulate with four values of $\lambda=0.0125,0.025,0.0375,0.05$ at the SU(3)-flavor symmetric point $\left(\kappa_{l}=\kappa_{s}=0.120900\right)$. Figure 1 shows results for the ground state proton energy as a function of $\lambda$ for both spin-up (positive $\lambda$ ) and -down (negative $\lambda$ ) states. In the two data sets, the $\lambda$ term in Eq. (11) has been added to the up quark and down quark separately. We fit to a Taylor expansion in the parameter $\lambda$,

$$
E(\lambda)=E(0)+\lambda \Delta q+\left.\frac{1}{2} \lambda^{2} \frac{\partial^{2} E(\lambda)}{\partial \lambda^{2}}\right|_{\lambda=0}+\ldots
$$

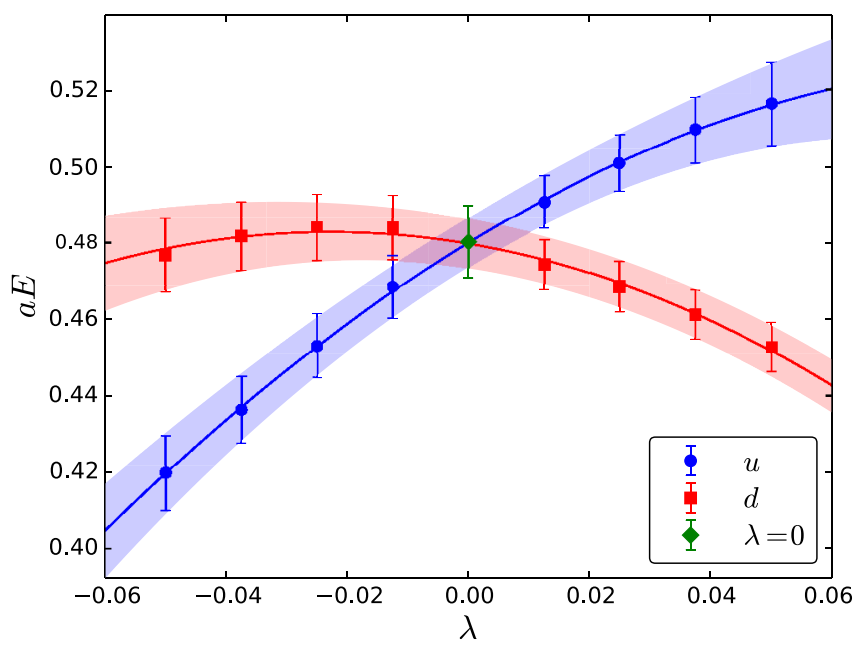

FIG. 1 (color online). Change in proton energy with the parameter $\lambda$. The two data sets show the effect when the extra term is applied to each light flavor. Note that at the $\lambda=0$ point we have the unshifted proton energy averaged over both spin projections. $\kappa_{l}=\kappa_{s}=0.120900$. 
retaining only up to quadratic terms in this case. We see that the slopes of the energy as a function of $\lambda$ for the two flavors of quark have opposite signs, indicating the expected result that the up quark has a positive spin contribution, and the down quark a negative contribution. We also note the presence of quadratic and higher order terms in $\lambda$ at larger $\lambda$. These are not presently of interest, as only the linear behavior at $\lambda=0$ is required to apply Eq. (14).

Using the linear parameter from the fit in Eq. (18), we have for the (unrenormalized) connected quark spin contributions in the proton,

$$
\begin{gathered}
\Delta u_{\mathrm{conn}}^{\mathrm{latt}}=0.97(13), \\
\Delta d_{\mathrm{conn}}^{\mathrm{latt}}=-0.27(11) .
\end{gathered}
$$

The errors here come from a bootstrap analysis of the proton correlators. Noting the form of the interpolating operator in Eq. (15), specifically the quark content, we may interchange up and down quarks above to obtain symmetric results for the proton's isospin partner, the neutron. Henceforth we will not distinguish between individual members of isospin multiplets when quoting results (all calculations are performed in the isospin symmetric limit). Specific quark flavors can be deduced from the context.

\section{B. Correlator ratios}

By taking advantage of the correlation between results at different $\lambda$ using the same statistical ensemble, we may dramatically improve the previous results. We can write the energy of a general hadron in terms of an energy shift $\Delta E(\lambda)$ as

$$
E(\lambda)=E(\lambda=0)+\Delta E(\lambda)
$$

where $E_{0}=M$ is the mass of the hadron. Then Eq. (14) becomes

$$
\Delta q_{\mathrm{conn}}^{\mathrm{latt}}=\left.\frac{\partial \Delta E(\lambda)}{\partial \lambda}\right|_{\lambda=0}
$$

Hence we only need to calculate energy shifts with respect to $\lambda$ in order to make use of the Feynman-Hellmann theorem. These energy shifts can be determined accurately from ratios of two-point functions.

For large times $t$ we expect that a lattice two-point function has the asymptotic form

$$
C(\lambda, t) \stackrel{\text { large } t}{\longrightarrow} \frac{e^{-E(\lambda) t}}{2 E(\lambda)}|A(\lambda)|^{2}
$$

Considering the ratio of two such correlation functions, one calculated with $\lambda=0$ and the other at $\lambda \neq 0$, we have

$$
\frac{C(\lambda, t)}{C(\lambda=0, t)} \stackrel{\text { large } t}{\longrightarrow} e^{-\Delta E(\lambda) t} \frac{E(0)}{E(\lambda)} \frac{|A(\lambda)|^{2}}{|A(0)|^{2}} .
$$

The exponential dependence of the above ratio of correlators contains the difference in energies between the undisturbed energy and the energy at some $\lambda$. Using this quantity to measure energy shifts allows us to make use of correlations between calculations with different values of $\lambda$. Since each calculation is performed using the same set of underlying gauge configurations, we expect fluctuations in the correlators to largely cancel, leaving a much cleaner signal.

Returning to the example of the last section, the upper plot in Fig. 2 shows nucleon effective mass plots for different values of $\lambda$ on the up quark, and the fit-range used
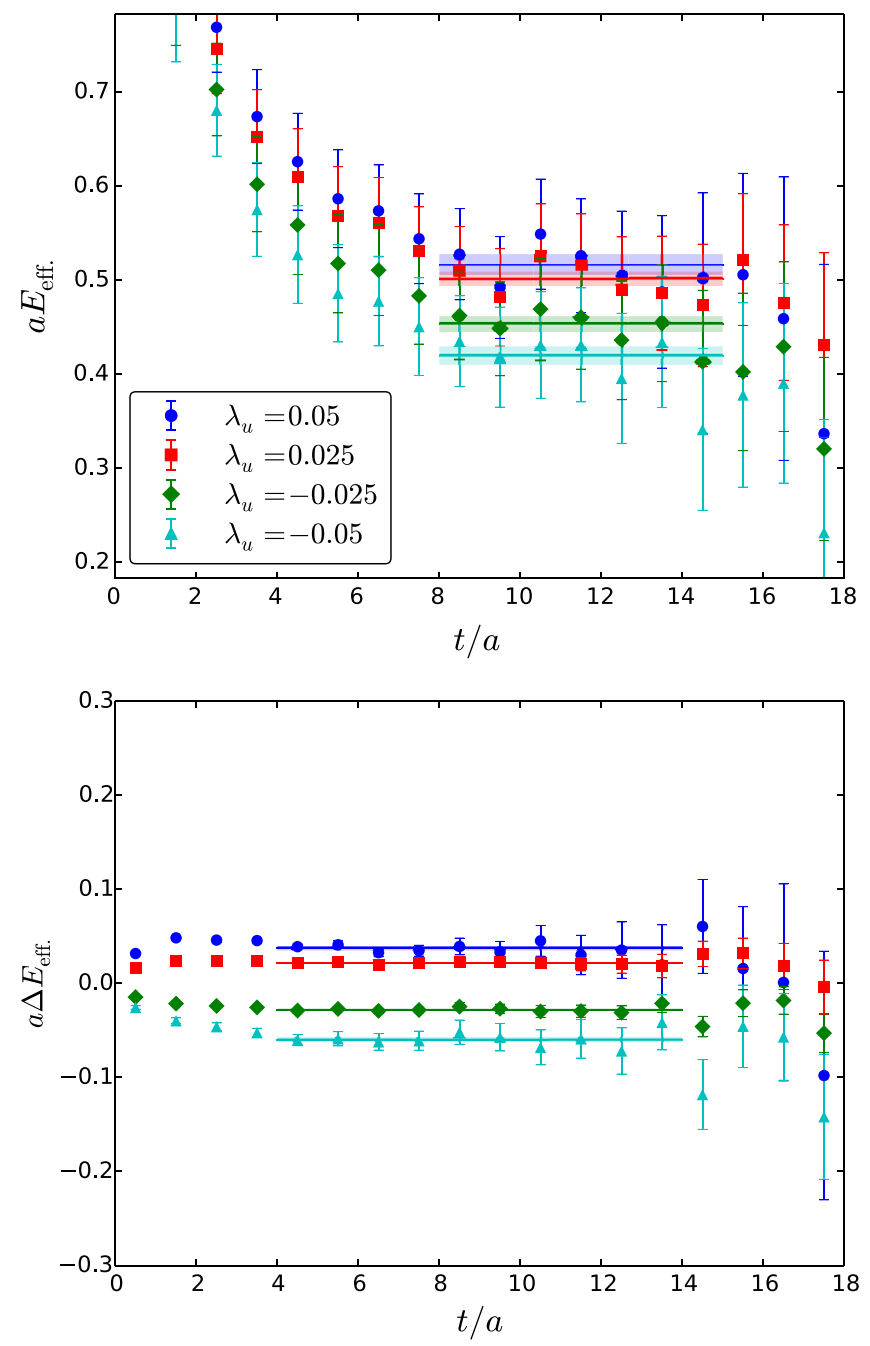

FIG. 2 (color online). Nucleon effective mass plots for different values of $\lambda$ on the up quark at the SU(3)-flavor symmetric point. The first plot shows absolute energies, and the second energy shifts obtained from correlator ratios. Only a few $\lambda$ have been included for clarity. The vertical scale is the same for both plots (only shifted), emphasizing the improvement achieved. 
for each. The lower plot displays the effective masses for the energy shifts obtained from the ratio of correlators in Eq. (24). We note that the energy shifts are much clearer using the new procedure, and we are able to fit at earlier times, possibly due to the canceling of excited states. Figure 3 shows the resulting nucleon energy shifts as a function of $\lambda$. We observe that the relative errors between different points are now much smaller, and we are able to much more tightly constrain the quadratic fit as compared to Fig. 1. We are also able to fix the $\lambda=0$ point to zero, since there is no energy shift for zero background spinfield. The linear plus quadratic behavior seen previously has been preserved, as we have only shifted all data points by a constant amount.

From the linear parameter in the quadratic fit, we calculate the quark axial charges

$$
\begin{gathered}
\Delta u_{\mathrm{conn}}^{\mathrm{latt}}=0.990(20), \\
\Delta d_{\mathrm{conn}}^{\mathrm{latt}}=-0.313(14) .
\end{gathered}
$$

These results are consistent with those in Eqs. (19) and (20), and our uncertainties have been significantly reduced. Note that if we instead extract the linear parameter from a fit including also a cubic term in Eq. (18), we find no change within the quoted statistical error, suggesting that retaining terms up to quadratic order in $\lambda$ is sufficient.

For comparison we have results calculated with a threepoint function method [32] using 330 configurations from the same larger ensemble of 1500 configurations from which the 350 used in this work were sourced. The threepoint functions method gives for the quark axial charges,

$$
\begin{gathered}
\Delta u_{\mathrm{conn}}^{\mathrm{latt}}=0.911(29), \\
\Delta d_{\mathrm{conn}}^{\mathrm{latt}}=-0.290(16),
\end{gathered}
$$

where we see comparable precision with our results in Eqs. (25) and (26), but obvious tension with the result for

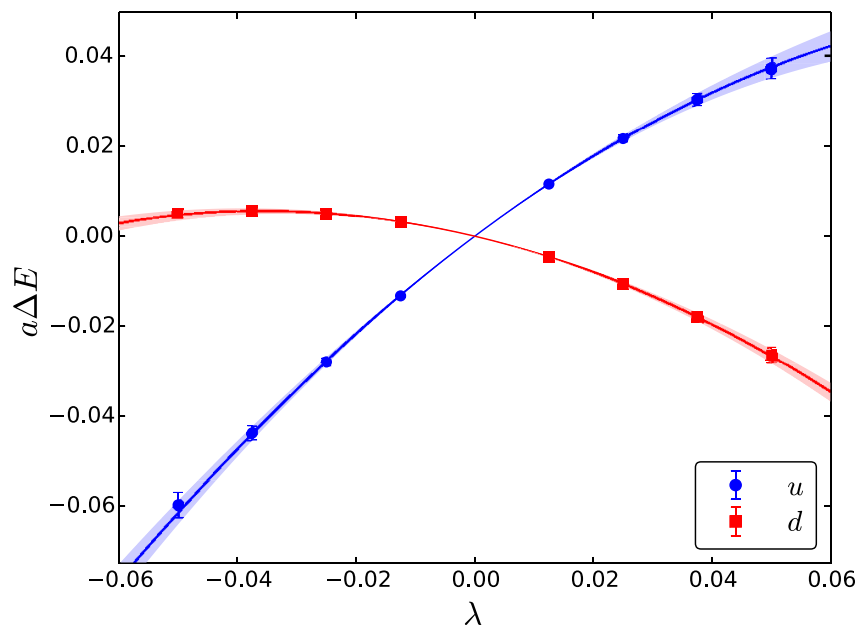

FIG. 3 (color online). Change in nucleon energy for different parameter values with a quadratic fit. $\kappa_{l}=\kappa_{s}=0.120900$. the up quark. This may be due to the fact that the FeynmanHellmann method has a greater control of excited-state contamination than the fixed-sink three-point method with a single source-sink smearing.

We note that future simulations investigating disconnected contributions will require the generation of separate sets of gauge fields for each value of $\lambda$ realized. The lack of correlation between such ensembles means one cannot take advantage of correlator ratios as described to reduce statistical noise. However, one could take correlated ratios of spin-up and spin-down correlation functions from the same underlying set of gauge field configurations to achieve a similar end.

\section{Optimization}

The spin matrix elements studied here have utilized numerous values of the background field strength $\lambda$ in order to accurately determine the derivative in the zero-field limit. As each value of the background field parameter requires the computation of a new set of propagators, we explore how one could best optimize the signal strength for a minimal set of inversions. This optimization is particularly necessary in the context of extending this work to disconnected operators, where new additional simulations are required for each value of the field strength.

We would like to realize a minimum of two different field strengths (with spin-up or -down projections), and restrict ourselves to a fixed-intercept quadratic fit in $\lambda$. Quadratic terms do not affect the linear terms that we are interested in, because these terms shift the energies equally on either side of the $\lambda=0$ point. Realizing a minimum of two field strengths (four values of $\lambda$, after spin-up or -down projection) allows us to be confident in uncertainties calculated for the two-parameter fixed-intercept quadratic fit.

We consider fitting quadratically to subsets of our existing results at the SU(3)-flavor symmetric point $\left(\kappa_{l}=\right.$ $\left.\kappa_{s}=0.120900\right)$, realizing only two of the four values of $\lambda$ at a time (four total data points after spin projection). Table II shows results for the quark axial charges calculated using these subsets. The calculated axial charges remain consistent within errors with each different fit; however, there

TABLE II. Connected spin contributions to the proton calculated using partial fits to only two values of the external field strength, $\lambda$. The fit used is given in Eq. (18), where we retain up to quadratic terms. The first column lists the values of $\lambda$ used, and the second shows the calculated values of the quark axial charges that result.

\begin{tabular}{lccc}
\hline \hline$\lambda_{1}$ & $\lambda_{2}$ & $\Delta u_{\text {conn }}^{\text {latt }}$ & $\Delta d_{\text {conn }}^{\text {latt }}$ \\
\hline 0.0125 & 0.0250 & $0.994(18)$ & $-0.313(13)$ \\
0.0125 & 0.0375 & $0.992(19)$ & $-0.312(13)$ \\
0.0125 & 0.0500 & $0.988(19)$ & $-0.311(14)$ \\
0.0250 & 0.0375 & $0.991(21)$ & $-0.314(14)$ \\
0.0250 & 0.0500 & $0.987(23)$ & $-0.313(15)$ \\
0.0375 & 0.0500 & $0.981(27)$ & $-0.314(17)$ \\
\hline \hline
\end{tabular}


does seem to be a systematic shift in $\Delta u$ as we move to higher $\lambda$. Also, the statistical errors in the energy shifts increase as $\lambda$ increases. Importantly, we note that the results obtained from a quadratic fit to the smallest two values of $\lambda$ (in the top row of Table II) agree within errors with results obtained from a fit to the entire data set in Eqs. (25) and (26), and with comparable statistical error. For these reasons, we restrict ourselves to realizing only the two smallest $\lambda$ when considering our second ensemble at smaller pion mass, as this appears to give sufficient accuracy and precision.

Also, we note that using only two values of the background field strength brings the total number of matrix inversions required for a computation of the axial charge of the proton in line with the standard three-point function method. With two values of $\lambda$, three colors and four spinor indices, we must calculate 36 inversions for every operator that we wish to investigate. For a three-point function calculation, three colors, four spinor indices and three quark propagators also lead to 36 inversions for each hadron that we consider. If the aim is to compute the forward proton matrix element of the axial operator, our results indicate that the Feynman-Hellmann method can achieve comparable statistical precision to the three-point function approach, at fixed computational cost.

\section{RESULTS}

Here we summarize connected quark spin contributions obtained using correlator ratio methods for the octet and decuplet baryons and vector mesons. All results quoted are from quadratic fits in $\lambda$. Calculations at the SU(3) symmetric point make use of the full data set of four values of $\lambda$. Simulations carried out away from the SU(3)-flavor symmetric point realize two values of the background field strength parameter, $\lambda=0.0125,0.025$, as motivated by the discussion in Sec. IV C. For all analyses we make use of correlator ratios as discussed in Sec. IV B.

\section{A. Octet baryons $\left(J=\frac{1}{2}\right)$}

Using the preliminary renormalization in Eq. (10), we conclude our discussion of the proton in Sec. IV by quoting renormalized values for $g_{A}$ at our two simulated pion masses,

$$
\begin{aligned}
& g_{A}\left(m_{\pi}=470 \mathrm{MeV}\right)=1.105(29), \\
& g_{A}\left(m_{\pi}=360 \mathrm{MeV}\right)=1.072(32),
\end{aligned}
$$

which are in good agreement with results in the literature, [16-21] (or [33] for a recent review). For the remaining octet baryons (excluding the $\Lambda$ ) we re-use the form of the interpolating operator for the proton in Eq. (15),

$$
\mathcal{O}_{\text {octet }}=\epsilon_{a b c}\left(q_{1 a}^{T} C \gamma_{5} q_{2 b}\right) q_{1 c},
$$

substituting light and strange quarks to access the $\Sigma$ and $\Xi$ states (in the isospin-symmetric limit). For example for the $\Sigma^{+}$we use the operator

$$
\mathcal{O}_{\Sigma^{+}}=\epsilon_{a b c}\left(u_{a}^{T} C \gamma_{5} s_{b}\right) u_{c} \text {. }
$$

In addition, we use the spin and parity-projection operators given in Eqs. (16) and (17).

The calculation proceeds as described in Sec. IV, and Table III shows results for the octet (details of the $\Lambda$ calculation are discussed later). $q_{1}$ and $q_{2}$ in the table refer to the quark flavors as they appear in the appropriate form of the interpolating operator in Eq. (31). As we are working in the isospin-symmetric limit, the results quoted can be applied to all members of each isospin multiplet, with appropriate flavor relabeling. So for instance we have for the $\Sigma^{+}$(quark content uus) that $\Delta q_{1}=\Delta u$ and $\Delta q_{2}=\Delta s$, whereas for the $\Xi^{0}$ (quark content uss) $\Delta q_{1}=\Delta s, \Delta q_{2}=\Delta u$.

Away from the SU(3) symmetric point (at the lighter pion mass) we see evidence for SU(3)-flavor-breaking effects in the quark spin contributions to the baryon octet. As we discussed in Sec. III, the singlet quark mass is the same for both ensembles, so the light quarks are lighter and the strange quark heavier on the second ensemble. We see $\Delta u$ and $\Delta d$ decreasing for the nucleon, whereas $\Delta u(\Delta d)$ decreases and $\Delta s$ increases for the $\Xi^{0(-)}$.

By comparing the individual quark flavor results of the octet baryons, we can gain an insight into the environmental sensitivity of the quark axial charges. As we move from the $\mathrm{N}$ to $\Sigma$ state with the heavier strange quark for example, we see the light quark contribution decreasing.

For the $\Lambda$ baryon we use the interpolating operator

$$
\begin{aligned}
\mathcal{O}_{\Lambda}= & \epsilon_{a b c} \frac{1}{\sqrt{6}}\left[2\left(u_{a}^{T} C \gamma_{5} d_{b}\right) s_{c}+\left(u_{a}^{T} C \gamma_{5} s_{b}\right) d_{c}\right. \\
& \left.-\left(d_{a}^{T} C \gamma_{5} s_{b}\right) u_{c}\right] .
\end{aligned}
$$

Note that when calculating two-point functions for the $\Lambda$, we do not calculate separate propagators for the up and down quarks. Hence the spin-field term in Eq. (11) is added to both light quarks at once, and so in Table III, $\Delta q_{1}=$ $\Delta u+\Delta d$ and $\Delta q_{2}=\Delta s$. Figure 4 shows results for the energy shift of the $\Lambda$ baryon on the $\kappa_{l}=0.121040, \kappa_{s}=$ 0.120620 ensemble for two values of $\lambda=0.0125,0.025$.

TABLE III. Table of connected spin contributions for the baryon octet. For all baryons except the $\Lambda, q_{1}$ and $q_{2}$ are as they appear in Eq. (31). For the $\Lambda, \Delta q_{1}=\Delta u+\Delta d$ and $\Delta q_{2}=\Delta s$.

\begin{tabular}{lrrrrr}
\hline \hline$\kappa_{l}$ & \multicolumn{2}{c}{0.120900} & & \multicolumn{2}{c}{0.121040} \\
$\kappa_{s}$ & \multicolumn{2}{c}{0.120900} & & \multicolumn{2}{c}{0.120620} \\
\cline { 1 - 2 } \cline { 5 - 6 } \cline { 5 - 6 } & $\Delta q_{1 \text { conn }}^{\text {latt }}$ & $\Delta q_{2 \text { conn }}^{\text {latt }}$ & & $\Delta q_{1 \text { conn }}^{\text {latt }}$ & $\Delta q_{2 \text { conn }}^{\text {latt }}$ \\
\hline $\mathrm{N}$ & $0.990(20)$ & $-0.313(14)$ & & $0.971(22)$ & $-0.291(20)$ \\
$\Sigma$ & $0.990(20)$ & $-0.313(14)$ & & $0.948(18)$ & $-0.297(8)$ \\
$\Xi$ & $0.990(20)$ & $-0.313(14)$ & & $1.039(12)$ & $-0.275(11)$ \\
$\Lambda$ & $-0.070(23)$ & $0.785(18)$ & $-0.050(17)$ & $0.803(10)$ \\
\hline \hline
\end{tabular}




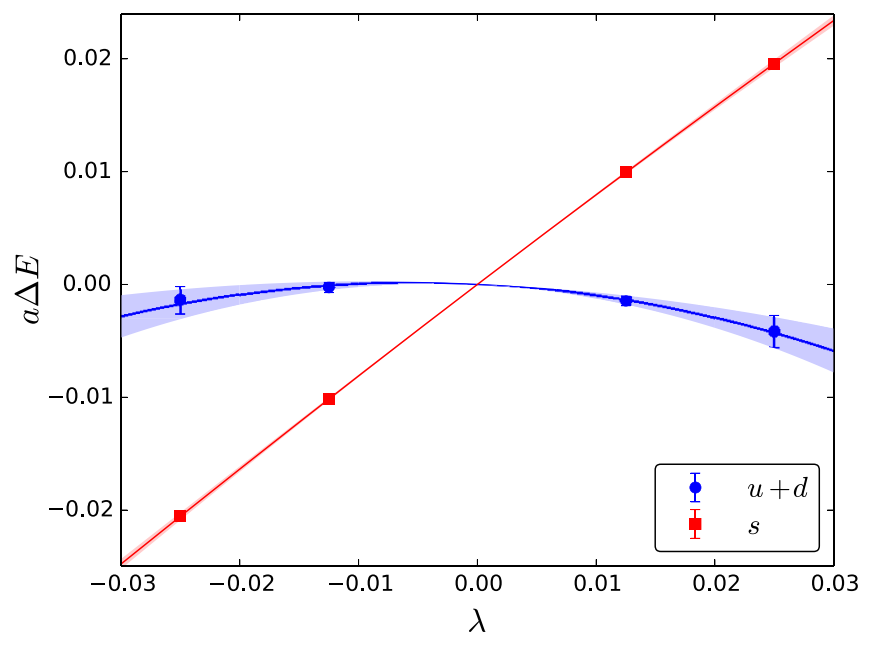

FIG. 4 (color online). Energy shift of the $\Lambda$ baryon with respect to $\lambda . \kappa_{l}=0.121040, \kappa_{s}=0.120620$.

The strong, highly constrained positive slope for the strange quark axial charge is consistent with the common expectation that the heavier strange quark carries the dominant spin fraction. Conversely, the small negative light quark contribution is more weakly constrained, subject predominantly only to quadratic effects.

In order to make a comparison with existing results in the literature, we make use of the preliminary results for $Z_{A}$ given in Eq. (10). For the $\Lambda$ baryon at the lighter pion mass of around $360 \mathrm{MeV}$, we have for light and strange connected contributions,

$$
\begin{gathered}
\Delta u_{\mathrm{conn}}=\Delta d_{\mathrm{conn}}=-0.043(14) \\
\Delta s_{\mathrm{conn}}=0.683(18) .
\end{gathered}
$$

Very few other lattice calculations of these quantities have been performed, the only example being in [34] from a chiral extrapolation of quenched calculations at pion masses of around $600 \mathrm{MeV}$ and upwards,

$$
\begin{gathered}
\Delta u_{\mathrm{conn}}=\Delta d_{\mathrm{conn}}=-0.02(4), \\
\Delta s_{\mathrm{conn}}=0.68(4),
\end{gathered}
$$

which are in good agreement.

\section{B. Decuplet baryons $\left(J=\frac{3}{2}\right)$}

For the decuplet baryons, we make use of the interpolating operator

$$
\begin{aligned}
\mathcal{O}_{\text {decuplet }}= & \epsilon_{a b c} \frac{1}{\sqrt{3}}\left[2\left(q_{1 a}^{T} C \gamma_{ \pm} q_{2 b}\right) q_{1 c}\right. \\
& \left.+\left(q_{1 a}^{T} C \gamma_{ \pm} q_{1 b}\right) q_{2 c}\right]
\end{aligned}
$$

where we define $\gamma_{ \pm}$(in Euclidean space) as

$$
\gamma_{ \pm}=-i \frac{1}{2}\left(\gamma_{1} \pm i \gamma_{2}\right)
$$

Analagously to the case of the octet baryons, appropriate quark flavors are substituted into Eq. (38) to access all decuplet states. We again make use of the parity and spinprojection operators in Eqs. (16) and (17). However, we must take care when analyzing the $m= \pm \frac{1}{2}$ states. Using the $\gamma_{ \pm}$matrices, we create diquarks with $J=1$ and $m= \pm 1$. The $\Gamma_{ \pm}$operator projects out the spin of the single quark to $m= \pm \frac{1}{2}$ as before. For the $m= \pm \frac{3}{2}$ baryon states, there is no problem combining the diquark and single quark, since

$$
\begin{aligned}
& |1+1\rangle\left|\frac{1}{2}+\frac{1}{2}\right\rangle=\left|\frac{3}{2}+\frac{3}{2}\right\rangle, \\
& |1-1\rangle\left|\frac{1}{2}-\frac{1}{2}\right\rangle=\left|\frac{3}{2}-\frac{3}{2}\right\rangle .
\end{aligned}
$$

However, when we create the $m= \pm \frac{1}{2}$ states, we create a mixture of $J=\frac{3}{2}$ and $J=\frac{1}{2}$ states,

$$
\begin{aligned}
& |1+1\rangle\left|\frac{1}{2}-\frac{1}{2}\right\rangle=\sqrt{\frac{1}{3}}\left|\frac{3}{2}+\frac{1}{2}\right\rangle+\sqrt{\frac{2}{3}}\left|\frac{1}{2}+\frac{1}{2}\right\rangle, \\
& |1-1\rangle\left|\frac{1}{2}+\frac{1}{2}\right\rangle=\sqrt{\frac{1}{3}}\left|\frac{3}{2}-\frac{1}{2}\right\rangle-\sqrt{\frac{2}{3}}\left|\frac{1}{2}-\frac{1}{2}\right\rangle .
\end{aligned}
$$

In principle, it is possible to project onto definite $J=\frac{3}{2}, \frac{1}{2}$ states (see [35]). However, we note that the $J=\frac{1}{2}, \Delta(1750)$ state has a higher mass than the $J=\frac{3}{2}, \Delta(1232)$ state, and so we expect the $\Delta(1232)$ to saturate the ground state at large Euclidean time, although there is the possibility of slightly more excited-state contamination than in the $m= \pm \frac{3}{2}$ cases.

Figure 5 shows results for the energy shift of the $\Delta$ baryon with $\lambda$. Only results for the up quark are shown, since the spin contribution for the down quark differs by a factor of a half in the isospin-symmetric limit. In contrast to the nucleon, all quarks in the $\Delta$ have positive contributions.

Table IV summarizes results for the decuplet baryons. Results at the heavier pion mass for the $m= \pm \frac{1}{2}$ states are unavailable, as the code to calculate these was not implemented at the time of those initial runs. Note that the distinction between the two different quark flavor spin contributions is a result of the form of the interpolating operator. So for example, the overall strange connected contribution to the $\Omega$ baryon, $\Delta s^{\frac{3}{2} m}=\Delta q_{1}^{\frac{3}{2} m}+\Delta q_{2}{ }^{\frac{3}{2} m}$.

Similarly to the results for the octet baryons in Table III, we see the effect of the changing quark masses on the axial charges. We observe the same pattern of environmental sensitivity as was evident when comparing the nucleon and $\Xi$ away from the SU(3) symmetric point; the heavier 
FEYNMAN-HELLMANN APPROACH TO THE SPIN ...

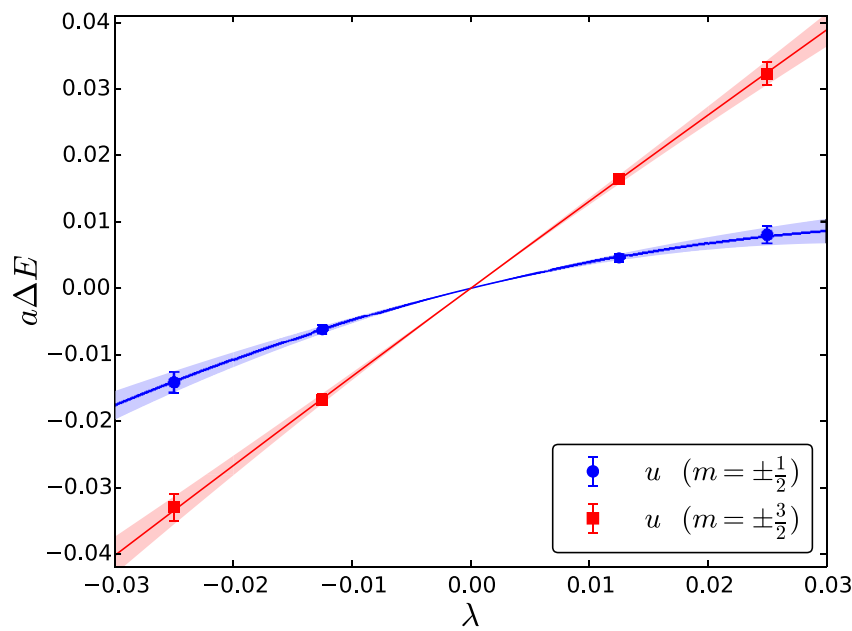

FIG. 5 (color online). Energy shift of $\Delta^{+}$with respect to $\lambda$ parameter on the $\kappa_{l}=0.121040, \kappa_{s}=0.120620$ ensemble. Only results for the up quark are shown (Results for the down quark differ by a factor of a half).

strange quark lowers the light quark contribution. For the zeroth moment [26],

$$
\Delta q^{\frac{3}{2} \frac{3}{2}}=3 \Delta q^{\frac{3}{2} \frac{1}{2}} .
$$

Comparing results for the $m= \pm \frac{1}{2}, \pm \frac{3}{2}$ states in Table IV, we see broad agreement with the sum rule.

Using the preliminary renormalization factor in Eq. (10), we have for the $\Delta$ baryon at the lighter pion mass of around $360 \mathrm{MeV}$,

$$
\Delta u_{\text {conn }}^{\frac{33}{2}}+\Delta d_{\text {conn }}^{\frac{33}{22}}=1.682(61),
$$

which compares well to results from [36] at a pion mass of 297(5) MeV,

$$
\Delta u_{\text {conn }}^{\frac{33}{2}}+\Delta d_{\text {conn }}^{\frac{33}{2}}=1.81(11) .
$$

TABLE IV. Table of connected spin contributions for the baryon decuplet. $q_{1}$ and $q_{2}$ are as they appear in the interpolating operator (38).

\begin{tabular}{lccccc}
\hline \hline$\kappa_{l}$ & \multicolumn{2}{c}{0.120900} & & \multicolumn{2}{c}{0.121040} \\
$\kappa_{s}$ & \multicolumn{2}{c}{0.120900} & & \multicolumn{2}{c}{0.120620} \\
\cline { 1 - 3 } \cline { 5 - 6 } \cline { 5 - 6 } & $\Delta q_{1 \text { conn }}^{\text {latt }}$ & $\Delta q_{2 \text { conn }}^{\text {latt }}$ & & $\Delta q_{1 \text { conn }}^{\text {latt }}$ & $\Delta q_{\text {2conn }}^{\text {latt }}$ \\
\hline$\Delta\left(m= \pm \frac{3}{2}\right)$ & $1.364(29)$ & $0.682(15)$ & & $1.319(48)$ & $0.660(24)$ \\
$\Sigma^{*}\left(m= \pm \frac{3}{2}\right)$ & $1.364(29)$ & $0.682(15)$ & & $1.310(43)$ & $0.727(11)$ \\
$\Xi^{*}\left(m= \pm \frac{3}{2}\right)$ & $1.364(29)$ & $0.682(15)$ & & $1.448(19)$ & $0.654(20)$ \\
$\Omega\left(m= \pm \frac{3}{2}\right)$ & $1.364(29)$ & $0.682(15)$ & & $1.437(16)$ & $0.718(8)$ \\
$\Delta\left(m= \pm \frac{1}{2}\right)$ & $\ldots$ & $\ldots$ & & $0.437(36)$ & $0.215(18)$ \\
$\Sigma^{*}\left(m= \pm \frac{1}{2}\right)$ & $\ldots$ & $\ldots$ & & $0.441(31)$ & $0.244(9)$ \\
$\Xi^{*}\left(m= \pm \frac{1}{2}\right)$ & $\ldots$ & $\ldots$ & & $0.506(14)$ & $0.215(14)$ \\
$\Omega\left(m= \pm \frac{1}{2}\right)$ & $\ldots$ & $\ldots$ & $0.504(12)$ & $0.248(6)$ \\
\hline \hline
\end{tabular}

PHYSICAL REVIEW D 90, 014510 (2014)

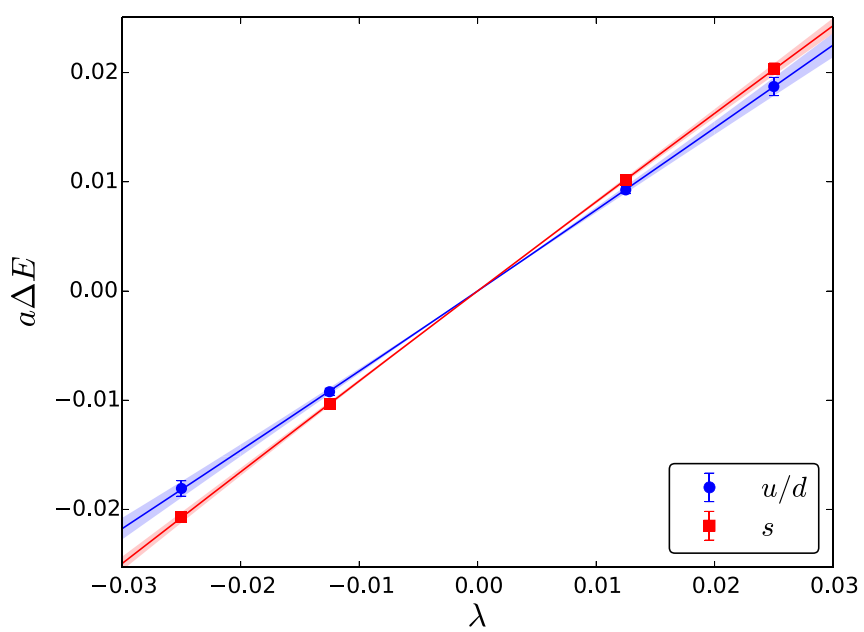

FIG. 6 (color online). Energy shift of $K^{*}$ with respect to $\lambda$ parameter on $\kappa_{l}=0.121040, \kappa_{s}=0.120620$ ensemble.

\section{Vector mesons $(J=1)$}

For the vector mesons, we make use of the interpolating operator

$$
\mathcal{O}_{\text {vector }}=\bar{q}_{2} \gamma_{ \pm} q_{1}
$$

where again appropriate quark flavors are substituted to access the different meson states.

Figure 6 shows results for the $K^{*}$ meson. We see that both quark flavors have positive contributions, and observe the slightly larger contribution from the strange quark. Table V summarizes results for the vector mesons.

We find relatively little change in the quark axial charges in the $\rho$ at the different quark masses (consistent with results reported in [37]). We do see a similar environmental sensitivity as in the octet and decuplet away from the SU(3) symmetric point. For example the strange spin contribution to the $K^{*}$ is greater than that for the $\phi$ due to the presence of the light quark in the $K^{*}$.

For the $\rho$ meson at the lighter pion mass of around $360 \mathrm{MeV}$, we have for the light spin contribution, using the preliminary renormalization in Eq. (10),

$$
\Delta u_{\mathrm{conn}}^{11}+\Delta d_{\mathrm{conn}}^{11}=1.311(64)
$$

\begin{tabular}{|c|c|c|c|c|}
\hline \multirow[t]{2}{*}{$\begin{array}{l}\kappa_{l} \\
\kappa_{s} \\
\end{array}$} & \multicolumn{2}{|c|}{$\begin{array}{l}0.120900 \\
0.120900\end{array}$} & \multicolumn{2}{|c|}{$\begin{array}{l}0.121040 \\
0.120620\end{array}$} \\
\hline & $\Delta q_{1 \text { conn }}^{\text {latt }}$ & $\Delta q_{2 \text { conn }}^{\text {latt }}$ & $\Delta q_{1 \text { conn }}^{\text {latt }}$ & $\Delta q_{2 \text { conn }}^{\text {latt }}$ \\
\hline$\rho$ & $0.762(14)$ & $0.762(14)$ & $0.771(33)$ & $0.771(33)$ \\
\hline$K^{*}$ & $0.762(14)$ & $0.762(14)$ & $0.738(22)$ & $0.821(15)$ \\
\hline$\phi$ & $0.762(14)$ & $0.762(14)$ & $0.793(11)$ & $0.793(11)$ \\
\hline
\end{tabular}

TABLE V. Table of connected spin contributions for the vector mesons. $q_{1}$ refers to the first flavor in Eq. (47), and $q_{2}$ to the second. 


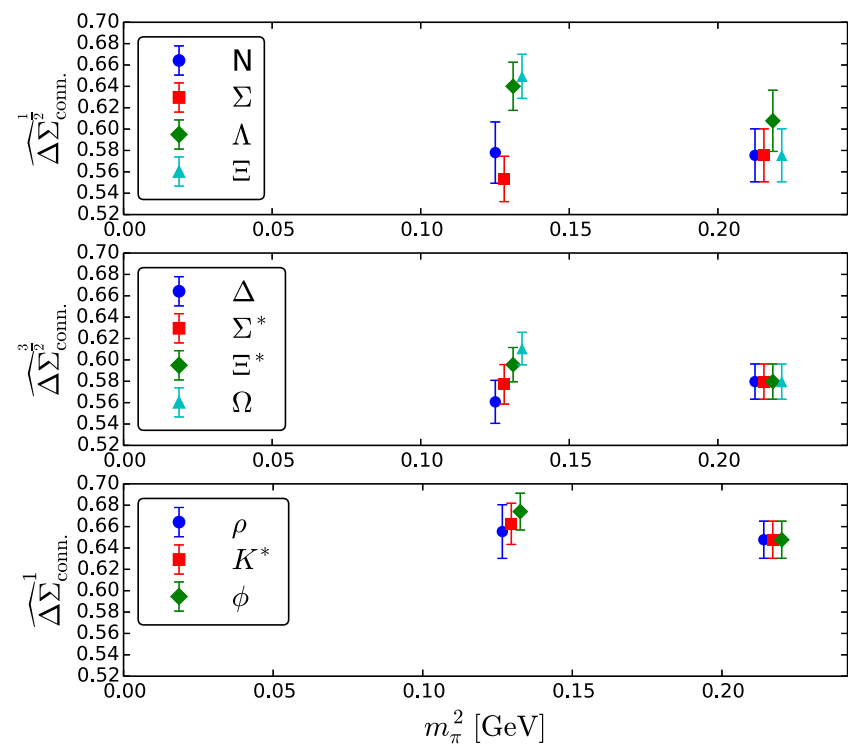

FIG. 7 (color online). The quark spin fraction $\widehat{\Delta \Sigma}_{\text {conn }}^{J}$ as defined in Eq. (50) for all the octet and decuplet baryons, and vector mesons, for different pion masses, renormalized using Eq. (10).

noting that the results quoted in Table $\mathrm{V}$ are for each light quark individually. Again, this calculation is rare in the literature. Ref. [37] quotes a value, after chiral extrapolation of quenched results, of

$$
\Delta u_{\mathrm{conn}}^{11}+\Delta d_{\mathrm{conn}}^{11}=1.180(92),
$$

where we see broad agreement with our results.

\section{Summary}

In order to compare the relative contributions of quarks to the spin of the different hadrons, we define the quark spin fraction for a spin- $J$ hadron to be

$$
\widehat{\Delta \Sigma}^{J}=\frac{\Delta \Sigma^{J}}{2 J} .
$$

Figure 7 shows $\widehat{\Delta \Sigma}_{\text {conn }}^{J}$ at different pion masses for all the baryons and mesons we have examined, renormalized using Eq. (10). Noting that the singlet quark mass is constant along our trajectory, we see that hadrons with dominant strange quark contributions have been shifted up with the increased strange mass, and hadrons with dominant light quark contributions have shifted down. We also see that the quark spin fraction for all the baryons studied here is in the range $55 \%-70 \%$.

\section{CONCLUDING REMARKS}

We have demonstrated that the Feynman-Hellmann method is an effective approach to calculating hadron matrix elements. We have demonstrated this through the determination of quark spin contributions to hadrons.
With the statistical improvements gained by examining hadron energy shifts, our calculations are able to achieve comparable precision to standard three-point function methods, with an equivalent computational investment. It is also possible that the Feynman-Hellmann method is less susceptible to excited-state contamination than these methods, a current point of debate within the lattice community.

We have also shown how the Feynman-Hellmann method may be most efficiently applied. In particular, it appears that only a couple of different background field strengths need be realized in order to make an accurate and precise calculation. Weaker field strengths give more tightly constrained fit parameters, and introduce less noise to correlation functions.

Our findings indicate that the possible application of the Feynman-Hellmann method to the calculation of such quantities as the disconnected quark spin contributions of hadrons is extremely promising. These simulations will require generating separate sets of gauge field configurations and a significant investment of computational time. However, the possibility of accessing such matrix elements so simply and with minimal excited-state contamination is extremely promising.

\section{ACKNOWLEDGEMENTS}

The numerical configuration generation was performed using the BQCD lattice QCD program, [38], on the IBM BlueGeneQ using DIRAC 2 resources (EPCC, Edinburgh, $\mathrm{UK}$ ), the BlueGene P and Q at NIC (Jülich, Germany) and the Cray XC30 at HLRN (The North-German Supercomputing Alliance). Some of the simulations were undertaken on the NCI National Facility in Canberra, Australia, which is supported by the Australian Commonwealth Government. The BLuEGENE codes were optimized using BAGEL [39]. The Chroma software library [40] was used in the data analysis. This investigation has been supported partly by the EU Grants No. 283286 (HadronPhysics3) and No. 227431 (Hadron Physics2) and by the Australian Research Council under Grants No. FT120100821, No. FT100100005, and No. DP140103067 (R. D. Y. and J. M. Z.).

\section{APPENDIX: A THE FEYNMAN-HELLMANN THEOREM}

Deriving the Feynman-Hellmann theorem in a fieldtheoretic sense is slightly different to the standard quantum mechanical approach found in textbooks. There are some constructions that must first be introduced, and we proceed by examining both two-point and three-point correlation functions.

\section{Lattice correlation functions}

We begin our discussion with the standard definitions of lattice two-point and three-point functions. Suppose, 
without loss of generality, that we have chosen $\bar{\chi}$ and $\chi$ as creation and annihilation operators for some hadron, such as the nucleon. For the Fourier transformed two-point function we have

$$
\int \mathrm{d}^{3} x e^{-i \vec{k} \cdot \vec{x}}\langle\chi(\vec{x}, t) \bar{\chi}(0)\rangle=\sum_{n} \frac{e^{-E_{n}(\vec{k}) t}}{2 E_{n}(\vec{k})}|\langle\Omega|\chi(0)| n, \vec{k}\rangle|^{2},
$$

where the right-hand side includes a sum over the excited states created by the chosen operators, and $|\Omega\rangle$ denotes the vacuum. This expression may be obtained using transfer matrix methods. At large Euclidean times, the summation is dominated by the ground state contribution,

$$
\int \mathrm{d}^{3} \vec{x} e^{-i \vec{k} \cdot \vec{x}}\langle\chi(\vec{x}, t) \bar{\chi}(0)\rangle \stackrel{\text { large } t}{\longrightarrow} \frac{e^{-E_{0}(\vec{k}) t}}{2 E_{0}(\vec{k})}|\langle\Omega|\chi(0)| 0, \vec{k}\rangle|^{2} .
$$

For the Fourier-transformed three-point function, we have

$$
\begin{aligned}
\int \mathrm{d}^{3} \vec{x} \mathrm{~d}^{3} \vec{y} e^{-i \vec{k} \cdot \vec{x}}\langle\chi(\vec{x}, t) \mathcal{O}(\vec{y}, \tau) \bar{\chi}(0)\rangle \\
=\sum_{n, m} \frac{e^{-E_{n}(\vec{k})(t-\tau)}}{2 E_{n}(\vec{k})} \frac{e^{-E_{m}(\vec{k}) \tau}}{2 E_{m}(\vec{k})}\langle\Omega|\chi(0)| n, \vec{k}\rangle \\
\quad \times\langle n, \vec{k}|\mathcal{O}(0)| m, \vec{k}\rangle\langle m, \vec{k}|\bar{\chi}(0)| \Omega\rangle,
\end{aligned}
$$

where we constrain ourselves here to the special case of zero momentum transfer between initial and final states. Integrating both sides of this expression with respect to $\tau$, we have

$$
\begin{aligned}
& \int_{0}^{t} \mathrm{~d} \tau \int \mathrm{d}^{3} \vec{x} \mathrm{~d}^{3} \vec{y} e^{-i \vec{k} \cdot \vec{x}}\langle\chi(\vec{x}, t) \mathcal{O}(\vec{y}, \tau) \bar{\chi}(0)\rangle \\
& =\sum_{n, m} \frac{e^{-E_{m}(\vec{k}) t}-e^{-E_{n}(\vec{k}) t}}{4 E_{n}(\vec{k}) E_{m}(\vec{k})\left(E_{n}(\vec{k})-E_{m}(\vec{k})\right)} \\
& \quad \times\langle\Omega|\chi(0)| n, \vec{k}\rangle\langle n, \vec{k}|\mathcal{O}(0)| m, \vec{k}\rangle\langle m, \vec{k}|\bar{\chi}(0)| \Omega\rangle .
\end{aligned}
$$

Now consider the large $t$ behavior of the right-hand side of this equation. When we expand the sums over $n$ and $m$, the dominant terms at large $t$ will be those with the lowest values of $E_{n}$ and $E_{m}$, when $n=m=0$. However, note that when $E_{n}=E_{m}$, the right-hand side is ill defined. So we first take the limit as $E_{m} \rightarrow E_{n}$ using l'Hôpital's rule. Dropping explicit momentum dependence momentarily, we have that

$$
\lim _{E_{m} \rightarrow E_{n}} \frac{e^{-E_{m} t}-e^{-E_{n} t}}{4 E_{n} E_{m}\left(E_{n}-E_{m}\right)}=\frac{t e^{-E_{n} t}}{4 E_{n}^{2}} .
$$

The large $t$ behavior of Eq. (A4) is then given by

$$
\begin{aligned}
& \int_{0}^{t} \mathrm{~d} \tau \int \mathrm{d}^{3} \vec{x} \mathrm{~d}^{3} \vec{y} e^{-i \vec{k} \cdot \vec{x}}\langle\chi(\vec{x}, t) \mathcal{O}(\vec{y}, \tau) \bar{\chi}(0)\rangle \\
& \stackrel{\text { large } t}{\longrightarrow} \frac{t e^{-E_{H}(\vec{k}) t}}{4 E_{H}^{2}(\vec{k})}|\langle\Omega|\chi(0)| H, \vec{k}\rangle|^{2}\langle H, \vec{k}|\mathcal{O}(0)| H, \vec{k}\rangle,
\end{aligned}
$$

where we denote the ground state of our hadron as $|H, \vec{k}\rangle$ and its energy to be $E_{H}$

\section{The Feynman-Hellmann theorem}

We now proceed with a proof of the Feynman-Hellmann theorem, expanding upon a proof presented in [14]. Suppose that we modify the action $S$ of our theory in some way, such that it now depends on some parameter $\lambda$,

$$
S \rightarrow S(\lambda)
$$

Consider the two-point correlation function discussed in the previous section. In the path integral formalism, this correlator is given by

$$
\langle\chi(\vec{x}, t) \bar{\chi}(0)\rangle_{\lambda}=\frac{1}{Z(\lambda)} \int \mathcal{D} A \mathcal{D} \psi \mathcal{D} \bar{\psi} \chi(\vec{x}, t) \bar{\chi}(0) e^{-S(\lambda)},
$$

where the subscript $\lambda$ indicates that the correlator is to be evaluated with respect to the modified action, and we note that the partition function is now also a function of $\lambda$,

$$
Z(\lambda)=\int \mathcal{D} A \mathcal{D} \psi \mathcal{D} \bar{\psi} e^{-S(\lambda)}
$$

Taking the derivative with respect to $\lambda$ of both sides of Eq. (A8), it is straightforward to show that

$$
\begin{aligned}
\frac{\partial}{\partial \lambda}\langle\chi(\vec{x}, t) \bar{\chi}(0)\rangle_{\lambda}= & \left\langle\frac{\partial S(\lambda)}{\partial \lambda}\right\rangle_{\lambda}\langle\chi(\vec{x}, t) \bar{\chi}(0)\rangle_{\lambda} \\
& -\left\langle\chi(\vec{x}, t) \frac{\partial S(\lambda)}{\partial \lambda} \bar{\chi}(0)\right\rangle_{\lambda},
\end{aligned}
$$

noting that angular brackets here denote expectation values as given in the path-integral formalism, analagous to Eq. (A8),

$$
\langle\mathcal{O}\rangle=\frac{1}{Z} \int \mathcal{D} A \mathcal{D} \psi \mathcal{D} \bar{\psi} \mathcal{O} e^{-S}
$$

Fourier transforming both sides of Eq. (A10) and re-arranging terms, we obtain the expression 


$$
\begin{gathered}
\left\{\frac{\partial}{\partial \lambda}-\left\langle\frac{\partial S(\lambda)}{\partial \lambda}\right\rangle_{\lambda}\right\} \int \mathrm{d}^{3} \vec{x} e^{-i \vec{k} \cdot \vec{x}}\langle\chi(\vec{x}, t) \bar{\chi}(0)\rangle_{\lambda} \\
=-\int \mathrm{d}^{3} \vec{x} e^{-i \vec{k} \cdot \vec{x}}\left\langle\chi(\vec{x}, t) \frac{\partial S(\lambda)}{\partial \lambda} \bar{\chi}(0)\right\rangle_{\lambda} .
\end{gathered}
$$

Consider the first term on the left-hand side of this expression. We have a derivative with respect to $\lambda$ of the two-point correlator from Appendix A 1. Since our action now depends on the parameter $\lambda$, we have

$$
\begin{aligned}
& \int \mathrm{d}^{3} x e^{-i \vec{k} \cdot \vec{x}}\langle\chi(\vec{x}, t) \bar{\chi}(0)\rangle_{\lambda} \\
& =\sum_{n} \frac{e^{-E_{n}(\vec{k}, \lambda) t}}{2 E_{n}(\vec{k}, \lambda)}\left|\langle\Omega|\chi(0)| n, \vec{k}\rangle_{\lambda}\right|^{2},
\end{aligned}
$$

noting that the energy eigenvalues and amplitudes both depend on $\lambda$. In deriving this expression, we required that the vacuum state has zero energy. We note that in modifying our action, we may have shifted our vacuum energy to a nonzero value (for instance, if our modification to the action took the form of the operator $\lambda \bar{q} q$ for some parameter $\lambda$ ). However, we will assume that this is not the case, as the modifications we make to the action in the main body of the paper, namely the inclusion of the axial operator $\bar{q} i \gamma_{\mu} \gamma_{5} q$, do not shift the vacuum energy. We can calculate the derivative with respect to $\lambda$ of Eq. (A13),

$$
\begin{aligned}
\frac{\partial}{\partial \lambda} \int \mathrm{d}^{3} x e^{-i \vec{k} \cdot \vec{x}}\langle\chi(\vec{x}, t) \bar{\chi}(0)\rangle_{\lambda} \\
=\sum_{n} \frac{e^{-E_{n}(\vec{k}, \lambda) t}}{2 E_{n}(\vec{k}, \lambda)}\left\{-\left(t+\frac{1}{E_{n}(\vec{k}, \lambda)}\right) \frac{\partial E_{n}(\vec{k}, \lambda)}{\partial \lambda}+\frac{\partial}{\partial \lambda}\right\} \\
\quad \times\left|\langle\Omega|\chi(0)| n, \vec{k}\rangle_{\lambda}\right|^{2} .
\end{aligned}
$$

At large Euclidean times, the lowest energy state in the summation above will dominate the summation, and the term with linear time dependence will dominate the second and third terms. Hence we have

$$
\begin{aligned}
& \frac{\partial}{\partial \lambda} \int \mathrm{d}^{3} x e^{-i \vec{k} \cdot \vec{x}}\langle\chi(\vec{x}, t) \bar{\chi}(0)\rangle_{\lambda} \\
& \stackrel{\text { large } t}{\longrightarrow}-\frac{\partial E_{H}(\vec{k}, \lambda)}{\partial \lambda} \frac{t e^{-E_{H}(\vec{k}, \lambda) t}}{2 E_{H}(\vec{k}, \lambda)}\left|\langle\Omega|\chi(0)| H, \vec{k}\rangle_{\lambda}\right|^{2} .
\end{aligned}
$$

Next, consider the second term on the left-hand side of Eq. (A12),

$$
\left\langle\frac{\partial S(\lambda)}{\partial \lambda}\right\rangle_{\lambda} \int \mathrm{d}^{3} \vec{x} e^{-i \vec{k} \cdot \vec{x}}\langle\chi(\vec{x}, t) \bar{\chi}(0)\rangle_{\lambda} .
$$

The very first quantity is just a vacuum expectation value, and assuming that the modification of $S$ does not carry vacuum quantum numbers (to leading order in $\lambda$ ), this contribution will vanish. Finally, consider the term on the right-hand side of Eq. (A12),

$$
\int \mathrm{d}^{3} \vec{x} e^{-i \vec{k} \cdot \vec{x}}\left\langle\chi(\vec{x}, t) \frac{\partial S(\lambda)}{\partial \lambda} \bar{\chi}(0)\right\rangle_{\lambda}
$$

Defining the operator $\mathcal{O}$ such that

$$
\int \mathrm{d} \tau \int \mathrm{d}^{3} \vec{y} \mathcal{O}(\vec{y}, \tau)=\frac{\partial S(\lambda)}{\partial \lambda},
$$

we have exactly the three-point correlator described by Eq. (A6), noting however that the energies and amplitudes now have explicit $\lambda$ dependence. We also point out that while the implementation of the operator is made across the whole lattice, the correlation function will only receive a significant contribution between 0 and $t$. Hence, we restrict the $\tau$ integration to this domain, and have

$$
\begin{aligned}
& \int_{0}^{t} \mathrm{~d} \tau \int \mathrm{d}^{3} \vec{x} \mathrm{~d}^{3} \vec{y} e^{-i \vec{k} \cdot \vec{x}}\langle\chi(\vec{x}, t) \mathcal{O}(\vec{y}, \tau) \bar{\chi}(0)\rangle_{\lambda} \\
& \quad \stackrel{\text { large } t}{\longrightarrow} \frac{t e^{-E_{H}(\vec{k}, \lambda) t}}{4 E_{H}^{2}(\vec{k}, \lambda)}\left|\langle\Omega|\chi(0)| H, \vec{k}\rangle_{\lambda}\right|^{2}\langle H, \vec{k}|\mathcal{O}(0)| H, \vec{k}\rangle_{\lambda} .
\end{aligned}
$$

As above, we again assume the modification to the action has not shifted the vacuum energy. So starting from Eq. (A12) and taking the behavior at large $t$ on both sides, substituting in Eqs. (A15) and (A19) we have

$$
\begin{aligned}
- & \frac{\partial E(\vec{k}, \lambda)}{\partial \lambda} \frac{t e^{-E_{H}(\vec{k}, \lambda) t}}{2 E_{H}(\vec{k}, \lambda)}\left|\langle\Omega|\chi(0)| H, \vec{k}\rangle_{\lambda}\right|^{2} \\
= & -\frac{t e^{-E_{H}(\vec{k}, \lambda) t}}{4 E_{H}^{2}(\vec{k}, \lambda)}\left|\langle\Omega|\chi(0)| H, \vec{k}\rangle_{\lambda}\right|^{2}\langle H, \vec{k}|\mathcal{O}(0)| H, \vec{k}\rangle_{\lambda} .
\end{aligned}
$$

Canceling various factors, we obtain

$$
\frac{\partial E_{H}(\vec{k}, \lambda)}{\partial \lambda}=\frac{1}{2 E_{H}(\vec{k}, \lambda)}\langle H, \vec{k}|\mathcal{O}(0)| H, \vec{k}\rangle_{\lambda} .
$$

We can generalize this result to any hadron for which we can choose suitable interpolating operators. Additionally, the origin 0 was taken only as a convenient reference point. So in general for any hadron state $|H\rangle$, we have

$$
\frac{\partial E_{H}(\lambda)}{\partial \lambda}=\frac{1}{2 E_{H}(\lambda)}\langle H|\mathcal{O}| H\rangle_{\lambda} .
$$

This is our expression for the Feynman-Hellmann theorem in the context of field theory. 
[1] V. Y. Alexakhin et al. (COMPASS Collaboration), Phys. Lett. B 647, 8 (2007).

[2] M. Anselmino, A. Efremov, and E. Leader, Phys. Rep. 261, 1 (1995).

[3] B. W. Filippone and X.-D. Ji, Adv. Nucl. Phys. 26, 1 (2001).

[4] S. D. Bass, Rev. Mod. Phys. 77, 1257 (2005).

[5] C. A. Aidala, S. D. Bass, D. Hasch, and G. K. Mallot, Rev. Mod. Phys. 85, 655 (2013).

[6] C. Alexandrou, M. Constantinou, S. Dinter, V. Drach, K. Jansen, C. Kallidonis, and G. Koutsou, Phys. Rev. D 88, 014509 (2013).

[7] J. D. Bratt et al. (LHPC Collaboration), Phys. Rev. D 82, 094502 (2010).

[8] A. Sternbeck et al., Proc. Sci., LATTICE (2011) 177.

[9] S. N. Syritsyn et al., Proc. Sci., LATTICE (2011) 178.

[10] P. Hägler, Phys. Rep. 490, 49 (2010).

[11] G. S. Bali et al. (QCDSF Collaboration), Phys. Rev. Lett. 108, 222001 (2012).

[12] M. Deka et al., arXiv:1312.4816.

[13] W. Detmold, Phys. Rev. D 71, 054506 (2005).

[14] R. Horsley, R. Millo, Y. Nakamura, H. Perlt, D. Pleiter, P. E. L. Rakow, G. Schierholz, A. Schiller, F. Winter, and J. M. Zanotti (QCDSF/UKQCD Collaborations), Phys. Lett. B 714, 312 (2012).

[15] C. Alexandrou et al., Proc. Sci., LATTICE (2013) 289.

[16] B. J. Owen, J. Dragos, W. Kamleh, D. B. Leinweber, M. Selim Mahbub, B. J. Menadue, and J. M. Zanotti, Phys. Lett. B 723, 217 (2013).

[17] S. Capitani, M. Della Morte, G. von Hippel, B. Jäger, A. Jüttner, B. Knippschild, H. B. Meyer, and H. Wittig, Phys. Rev. D 86, 074502 (2012).

[18] B. Jäger et al., arXiv:1311.5804.

[19] S. Dinter, C. Alexandrou, M. Constantinou, V. Drach, K. Jansen, and D. B. Renner, Phys. Lett. B 704, 89 (2011).

[20] T. Bhattacharya, S. D. Cohen, R. Gupta, A. Joseph, H.-W. Lin, and B. Yoon, Phys. Rev. D 89, 094502 (2014).
[21] G. S. Bali et al., arXiv:1311.7041.

[22] A. W. Thomas, Adv. Nucl. Phys. 13, 1 (1984).

[23] G. A. Miller, Int. Rev. Nucl. Phys. 1, 189 (1984).

[24] A. W. Thomas, S. Theberge, and G. A. Miller, Phys. Rev. D 24, 216 (1981).

[25] P. E. Shanahan, A. W. Thomas, K. Tsushima, R. D. Young, and F. Myhrer, Phys. Rev. Lett. 110, 202001 (2013).

[26] R. L. Jaffe and A. Manohar, Nucl. Phys. B321, 343 (1989).

[27] R. Horsley et al., Proc. Sci., LATTICE (2013) 249.

[28] W. Bietenholz et al., Phys. Lett. B 690, 436 (2010).

[29] W. Bietenholz et al., Phys. Rev. D 84, 054509 (2011).

[30] M. Constantinou et al. (QCDSF) (to be published).

[31] S. Capitani, M. Göckeler, R. Horsley, H. Perlt, P. E. L. Rakow, G. Schierholz, and A. Schiller, Nucl. Phys. B593, 183 (2001).

[32] A. N. Cooke et al., Proc. Sci., LATTICE (2013) 278.

[33] S. Syritsyn, Proc. Sci., LATTICE (2014) 009.

[34] M. Göckeler, R. Horsley, D. Pleiter, P. E. L. Rakow, S. Schaefer, A. Schäfer, and G. Schierholz (QCDSF), Phys. Lett. B 545, 112 (2002).

[35] J. Zanotti, D. Leinweber, A. Williams, J. Zhang, W. Melnitchouk, and S. Choe (CSSM Lattice collaboration), Phys. Rev. D 68, 054506 (2003).

[36] C. Alexandrou, E. B. Gregory, T. Korzec, G. Koutsou, J. W. Negele, T. Sato, and A. Tsapalis, Phys. Rev. D 87, 114513 (2013).

[37] C. Best, M. Göckeler, R. Horsley, E.-M. Ilgenfritz, H. Perlt, P. Rakow, A. Schäfer, G. Schierholz, A. Schiller, and S. Schramm, Phys. Rev. D 56, 2743 (1997).

[38] Y. Nakamura and H. Stüben, Proc. Sci., LATTICE (2010) 040.

[39] P. A. Boyle, Comput. Phys. Commun. 180, 2739 (2009).

[40] R. G. Edwards and B. Joo (SciDAC Collaboration, LHPC Collaboration, UKQCD Collaboration), Nucl. Phys. B, Proc. Suppl. 140, 832 (2005). 